\title{
Analysis of Correlation Between Coronary Tortuosity and Calcification Score
}

\section{Min Li}

Zhongda Hospital, School of Medicine

\section{Zhen-Wei Wang}

Zhongda Hospital, School of Medicine

\section{Li-Juan Fang}

Zhongda Hospital, School of Medicine

\section{Shou-Quan Cheng}

Zhongda Hospital, School of Medicine

\section{Xin Wang}

Zhongda Hospital, School of Medicine

Nai-Feng Liu ( $\square$ liunf@seu.edu.cn )

Zhongda Hospital, School of Medicine

\section{Research Article}

Keywords: Coronary artery tortuosity, Coronary artery calcification, Atherosclerosis, Calcium score

Posted Date: September 21st, 2021

DOl: https://doi.org/10.21203/rs.3.rs-870867/v1

License: (c) (i) This work is licensed under a Creative Commons Attribution 4.0 International License.

Read Full License 


\section{Abstract}

Background: Coronary artery tortuosity (CAT) is regarded as a variation of vascular anatomy, and its relationship with coronary artery calcification (CAC) score is still not well clarified. Studying the correlation between coronary artery calcification scores and CAT to determine specific prevention and intervention populations seems to have more meaningful.

Methods: The study is a cross-sectional retrospective study, including 1280 patients with tortuous coronary artery. CAT is defined as the presence of at least three consecutive curvatures of more than $45^{\circ}$ measured during systole or diastole of a major epicardial coronary artery. Multivariable regression analysis was used to adjust the clinical parameters directly affecting CAT.

Results: Of these individuals, 445 (35\%) were evaluated having CAT, of which females are higher than males (59.1\% vs. $40.9 \%$ ). Moderate CAC score (101-400) (odds ratio (OR) 1.49, 95\% confidence interval [95\% Cl] 1.05-2.10, $P=0.025)$ revealed significantly associated with CAT on univariable analysis. However, multivariable analysis after adjusting for confounding factors only indicated that CAT was positively correlated with female (OR 1.60, 95\% Cl 1.22-2.10, $P=0.001$ ), hypertension (OR 1.34, 95\% $\mathrm{Cl} 1.03-1.74$, $P=0.028)$, and age (OR $1.04,95 \% \mathrm{Cl} 1.01-1.03, P=0.001)$, while was negatively associated with body mass index $(\mathrm{BMI})>28(\mathrm{OR} 0.48,95 \% \mathrm{Cl} 0.32-0.70, P<0.0001)$. Further analysis stratified by gender showed that compared with non-CAT, CAT was significantly linked with moderate CAC score (OR 1.79, 95\% Cl 1.003.20, $P=0.048$ ), hypertension (OR 1.54, 95\% Cl 1.07-2.22, $P=0.021$ ), and high-density lipoprotein (HDL) (OR $1.86,95 \% \mathrm{Cl} 1.07-3.24, P=0.028)$, while was negatively related to $\mathrm{BMI}>28$ (OR $0.51,95 \% \mathrm{Cl} 0.31-0.84$, $P<0.008)$ in female patients.

Conclusions: CAT is more likely to be found in females, connected with hypertension, age, and BMI. No significant correlation is found between the presence of tortuosity and calcium score or diameter stenosis on multivariable analysis. Whereas the CAT is associated with moderate CAC score in correlation analysis when women are selected as the main group.

\section{Introduction}

Vascular calcification is the active deposition process of bone-specific hydroxyapatite crystallization in the blood vessel wall caused by multiple pathogenic factors ${ }^{1}$. Vascular calcification is prevalent and is related to many diseases such as aging, diabetes, atherosclerosis and chronic kidney disease. Long-term vascular calcification can cause vessel stiffness and reduced compliance, leading to adverse cardiovascular events ${ }^{2}$. Raggi et al. documented that the progression of coronary calcification was more common in patients who had clinical coronary events compared with event-free subjects ${ }^{3}$. This view has also been confirmed by a multi-ethnic study of atherosclerosis conducted by Budoff et al., suggesting that vascular calcification is significantly associated with all-cause mortality and atherosclerotic plaque rupture ${ }^{4}$. Computed tomography angiography (CTA) provides a unique diagnostic value in the estimation 
of CAC progression, and it can be detected, located and quantified, which is of great significance to the risk assessment of coronary artery disease ${ }^{5}$.

Furthermore, CAT is a common phenomenon found in coronary angiography ${ }^{6}$. The etiology and clinical significance of CAT remain unclear ${ }^{7}$. Preliminary evidence suggests that it is associated with various diseases, including aging, hypertension, reversible myocardial reperfusion injury, spontaneous coronary artery dissection, fibromuscular dysplasia and connective tissue diseases ${ }^{8}$. Nevertheless, the correlation between CAT and atherosclerosis has been controversial, and few types of research have revealed its correlation with calcification score. It seems to be more beneficial to study the correlation between CAC score and vascular tortuosity in order to identify specific prevention and intervention populations.

\section{Patients And Methods}

\section{Clinical Study Design}

This is a cross-sectional retrospective study registered in the China Clinical Trial Registration Center (ChiCTR1800020259). The study included patients with suspected coronary artery disease and stable hemodynamics who underwent coronary CTA examinations using 320-row-detector dynamic volume CT from January 1, 2020 to March 9, 2021.The patients with a past history of coronary stent implantation, coronary artery bypass graft, prosthetic valve or pacemaker implantation, pregnancy, chronic renal insufficiency, and those presenting any contraindication for iodinated compounds were excluded.

\section{CT angiography}

Coronary CTA images were reviewed by two experienced radiologists who were blind to all clinical profiles of the participants. CAC score was calculated by dedicated software and quantified as the Agatston score ${ }^{9}$. According to the ACCF/AHA 2007 clinical expert consensus, the Agatston score was divided into 4 levels $(0,1-100,101-400,>400)^{10}$. In addition, the severity of coronary stenosis was classified as up to $0,1-24 \%, 25-49 \%, 50-69 \%, 70-100 \%$.

\section{Echocardiographic parameters}

One of two experienced operators blinded to all clinical and angiographic details performed the echocardiographic examination. Transthoracic echocardiography was done for all subjects using the GE Vivid E95 system (GE Vingmed Ultrasound, Horten, Norway) or the Philips EPIQ 7C (Philips Medical Systems, Andover, MA) ultrasound system. All echocardiographic images or parameters were obtained in accordance with the European Association of Echocardiography/American Society of Echocardiography (EAE/ASE). The inner diameters of aortic sinus (AO) and ascending aorta (AAO), left atrial anteroposterior diameter (LA), left or right ventricular end-diastolic inner diameter (LVEDd or RVEDd), left ventricular posterior wall thickness (LVPW), and interventricular septal thickness (IVS) were obtained in standard parasternal long axis view. Left ventricular ejection fraction (LVEF) was calculated by use of the arealength method. 


\section{Biochemical indexes}

Venous blood samples were collected from all participants after a 12-h overnight fast. Biochemical parameters were determined in the Department of Clinical Laboratory, Zhong Da hospital affiliated Southeast University.

\section{Risk factors}

Hypertension was defined as systolic blood pressure $\geq 140 \mathrm{mmHg}$ or/and diastolic blood pressure $\geq 90 \mathrm{mmHg}$, or taking antihypertensive drugs. Diabetes was diagnosed according to the WHO criteria. Smokers were defined as those smoking in the past 1 month.

\section{Statistical Analysis}

IBM SPSS software (version 24.0, SPSS, Inc.) was used for statistical analysis. All data were accomplished a normal distribution test before analysis. Continuous variables were expressed as

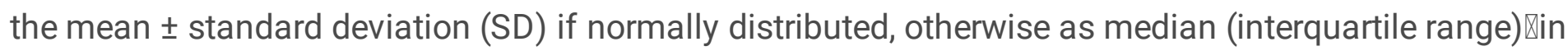
addition, comparisons between groups performed by the $t$ test if normally distributed or the MannWhitney test if not normally distributed. Categorical variables were described as percentages and compared by $\chi 2$ testing. Univariable and multivariable analysis were performed to identify clinical parameters directly affecting coronary tortuosity. All tests were 2-sided and $P$ values $<0.05$ were considered statistically significant.

\section{Results}

\section{Baseline characteristics}

Baseline characteristics of participants in the study were presented (Table 1). A total of 1280 subjects were included in the analysis, of whom 445 (35\%) were CAT group (Figure 1), and 835 (65\%) were N-CAT group. The mean \pm SD age of the participants was $62.3 \pm 12.7$ years. All variables, except gender $(P<0.0001)$, age $(P<0.0001)$, BMI $(P<0.0001)$, hypertension $(P=0.014)$, AO diameters $(P=0.006)$, LVEDd $(P=0.002)$, uric acid $(P=0.018)$, triglycerides (TG) $(P=0.047)$, and high-density lipoprotein (HDL) $(P<0.0001)$ were similar in both groups.

\section{No correlation between CAT and CAC score}

On univariable analysis, female was more likely to have CAT than male (OR 1.92, 95\% $\mathrm{Cl} 1.52-2.42$, $P<0.0001$ ) (Table 2 and Supplementary Table 1 ). The increase of age was also significantly related to CAT (OR 1.03, 95\% Cl 1.02-1.04, $P<0.0001)$. Moreover, participants with hypertension were more possible than those without to be CAT (OR 1.34, 95\% $\mathrm{Cl} 1.06-1.70, P=0.015)$. In addition, compared with patients without lumen stenosis, patients with moderate lumen stenosis were associated with CAT (OR 1.47, 95\% Cl 1.04-2.09, $P=0.029$ ), while individuals with an Agatston score from 101 to 400 were more likely to 
coexist with CAT than those with an Agatston score 0 (OR 1.49, 95\% Cl 1.05-2.10, $P=0.025$ ), although these trends were not statistically significant on multivariable analysis.

On multivariable analysis, after adjusting for the parameters that affect CAT, female (OR $1.60,95 \% \mathrm{Cl}$ 1.24-2.17, $P=0.001$ ), age (OR 1.02, 95\% Cl 1.01-1.03, $P=0.003$ ), and hypertension (OR 1.32, 95\% $\mathrm{Cl} 1.01$ $1.72, P=0.039$ ) remained significantly associated with CAT (Table 2). What was inconsistent was that on univariate analysis, overweight (BMI 24-27.9) (OR 0.71, 95\% Cl 0.55-0.92, $P=0.009)$ and obese patients (BMI>28) (OR 0.41, 95\% $\mathrm{Cl} 0.29-0.59, P<0.0001)$ were less likely to coexist with CAT than normal weight (BMI 18.5-23.9) (OR 0.47, 95\% $\mathrm{Cl} 0.32-0.70, P<0.0001)$ patients, while multivariate analysis only showed that obese patients were less likely to coexist with CAT than normal weight patients.

\section{Moderate calcification score is related to CAT in female patients}

In order to explore the correlation between coronary tortuosity and CAC score in special populations, gender was stratified and further analyzed. Univariate analysis revealed that in female patients, compared with patients with an Agatston score of 0 , patients with a high Agatston score were significantly associated with CAT (1-100, OR $1.49,95 \% \mathrm{Cl} 1.01-2.18, P=0.042 ; 101-400,0 \mathrm{R} 2.36,95 \% \mathrm{Cl}$ 1.36-4.07, $P=0.002$; $>400$, OR 1.82, 95\% Cl 1.07-3.11, $P=0.028$ ) (Table 3 and Supplementary Table 2), while this correlation was not found in male patients (Table 4 and Supplementary Table 2). However, on multivariate analysis, CAT in female patients was positively correlated with moderate Agatston score (OR 1.79, 95\% Cl 1.00-3.20, $P=0.048$ ), hypertension (OR 1.54, 95\% Cl 1.07-2.22, $P=0.021$ ) and HDL (OR $1.86,95 \% \mathrm{Cl} 1.07-3.24, P=0.028)$, and negatively correlated with obesity (OR $0.51,95 \% \mathrm{Cl} 0.31-0.84$, $P=0.008)$, while it in male patients was positively associated with age (OR $1.03,95 \% \mathrm{Cl} 1.02-1.04$, $P<0.0001)$ and negatively associated with overweight (OR $0.72,95 \% \mathrm{Cl} 0.56-0.94, P=0.014)$ and obesity (OR 0.46, 95\% $\mathrm{Cl} 0.32-0.66, P<0.0001$ ) (Table 3 and 4).

\section{Discussion}

In this study of 1280 participants, it is documented that age and hypertension may be the main factors for the occurrence of CAT, which is consistent with the results of other researchers ${ }^{6,11}$. It should be understood that arteries are usually straight tubes that can effectively transport blood to remote organs. However, due to developmental abnormalities or vascular disease, arteries may be tortuous. The maintenance of arterial stability in vivo mainly depends on a certain degree of axial tension, pressure and traction, and the retraction force produced by elastin which can resist pressure and traction ${ }^{12-15}$. These forces are essential in preventing tortuosity. The age-related coronary tortuosity is related to the degradation and decrease of elastin ${ }^{16-18}$; In addition, the axial tension may decrease with aging ${ }^{15,19}$. Hypertension or increased blood flow also contributes to artery tortuosity associated with elastin degradation and fragmentation, which has been considered the cause of vessel lengthening ${ }^{20,21}$.

The results of the study on the correlation between gender differences and CAT are also consistent with the findings of other current works ${ }^{11,22}$. It also explains why women often show symptoms of chest pain, 
but have better angiographic results being normal coronary arteries or less severe disease than men ${ }^{22}$. In addition, the uniqueness of this work is that it shows the correlation between BMI and CAT. Compared with people with normal weight, overweight or obesity is negatively correlated with CAT. Previous studies have reported that the incidence of tortuosity increased as the heart size and mass decreased ${ }^{23}$. Based on this theory, we speculate that long-term overweight and obesity are accompanied by an increase in the thickness of epicardial adipose tissue, which may have a certain impact on the shape and length of blood vessels.

We did not find a negative correlation between CAT and atherosclerosis described by Li et al. ${ }^{6}$, which may be due to different methods used to calculate the coronary tortuosity. Beyond that, our study displays no correlation between Agatston score and CAT except in the female population. Result contrasts with research by Tahlawi et al., who revealed that CAT is associated with Agatston score even in the absence of significant obstructive lesion 7 . This may be due to the large population cohort and detailed stratification of calcification score included in our study. Even so, the CAC score is highly specific in atherosclerosis ${ }^{24}$. However, the result that compared with patients without vascular calcification, those with moderate calcification score are associated with CAT in females is discovered. It is known that severe intravascular calcification can lead to the stiffness and rigidity of the vascular wall, which may be the reason for the insignificant difference in severe CAC score (Agatston score $>400$ ) between the two groups. Another interesting finding is that there is a significant correlation between HDL and CAT when female patients are analyzed independently. In this way, previous hypothesis that there was no significant difference between both groups regarding HDL level was overturned by us. HDL is known to exert an antiatherosclerotic effect by interacting with macrophages and other inflammatory immune cells ${ }^{25}$. As other studies have shown that CAT is inversely associated with atherosclerosis ${ }^{6}$. Perhaps a high level of HDL may be a potential protection mechanism.

\section{Limitations}

This study is a single-center cross-sectional study, which limits the comparability of our findings to the general population. In addition, there is a lack of classification in CAT severity, which is uniformly defined as at least three consecutive curvatures greater than 45 degrees in a major epicardial coronary artery. Moreover, there may be some differences between the two methods of coronary angiography and CTA in the assessment of coronary artery stenosis.

\section{Conclusions}

Tortuous arteries are common in humans. Although slight tortuosity is asymptomatic, severe tortuosity may cause an ischemic attack in remote organs. Our results show that tortuous arteries are related to aging, hypertension, $\mathrm{BMI}$, and gender. However, little is known about the mechanism of its formation and development. In the future, more research is needed to explore the mechanism and provide new technologies to prevent and treat vascular curvature. 


\section{Abbreviations}

CAT, coronary artery tortuosity; CAC, coronary artery calcification; OR, odds ratio; $95 \% \mathrm{Cl}$, confidence interval; $B M I$, body mass index; CTA, computed tomography angiography; $A O$, aortic sinus; AAO, ascending aorta; LA, left atrial anteroposterior diameter; LVEDd or RVEDd, left or right ventricular enddiastolic inner diameter; LVPW, left ventricular posterior wall thickness; IVS, interventricular septal thickness; LVEF, left ventricular ejection fraction; TG, triglycerides; HDL, high density lipoprotein; ApoA1, apolipoprotein A1; ApoB, apolipoprotein B; LPa, lipoprotein a.

\section{Declarations}

\section{Ethics approval and consent to participate}

This study was approved by the Ethical committee of Clinical Investigation of Southeast University School of Medicine (ZDSYLL066-P01). The research reported in this paper was in compliance with the ethical standards of the Declaration of Helsinki. Informed consents were taken from all individuals included in the study. All methods were performed in accordance with the relevant guidelines and regulations.

\section{Consent for publication}

Not applicable.

\section{Availability of data and materials}

All data are available without restriction. Researchers can obtain data by contacting the corresponding author.

\section{Competing interests}

All authors declare no conflict of interest.

\section{Funding}

The research receives funding from the National Nature Science Foundation of China (No. 81770451 and No. 81970381). The funders had no role in study design, data collection and analysis, decision to publish, or preparation of the manuscript.

\section{Authors' contributions}

Min Li: Writing-original draft. Zhen-Wei Wang: Data collection and verification. Li-Juan Fang: Writingreview and editing. Shou-Quan Cheng: Supervision. Nai-Feng Liu: Reviewing and Editing, Supervision.

\section{Acknowledgements}


Not applicable.

\section{References}

1. Nicoll, R and Henein, MY, The predictive value of arterial and valvular calcification for mortality and cardiovascular events, Int J Cardiol Heart Vessel, 2014;3:1-5.

2. Durham, AL, Speer, MY, Scatena, M, et al., Role of smooth muscle cells in vascular calcification: implications in atherosclerosis and arterial stiffness, Cardiovasc Res, 2018;114:590-600.

3. Raggi, P, Callister, TQ and Shaw, LJ, Progression of coronary artery calcium and risk of first myocardial infarction in patients receiving cholesterol-lowering therapy, Arterioscler Thromb Vasc Biol, 2004;24:1272-1277.

4. Budoff, MJ, Young, R, Lopez, VA, et al., Progression of coronary calcium and incident coronary heart disease events: MESA (Multi-Ethnic Study of Atherosclerosis), J Am Coll Cardiol, 2013;61:12311239.

5. Lehmann, N, Erbel, R, Mahabadi, AA, et al., Value of Progression of Coronary Artery Calcification for Risk Prediction of Coronary and Cardiovascular Events: Result of the HNR Study (Heinz Nixdorf Recall), Circulation, 2018;137:665-679.

6. Li, Y, Shen, C, Ji, Y, et al., Clinical implication of coronary tortuosity in patients with coronary artery disease, PLoS One, 2011;6:e24232.

7. El Tahlawi, M, Sakrana, A, Elmurr, A, et al., The relation between coronary tortuosity and calcium score in patients with chronic stable angina and normal coronaries by CT angiography, Atherosclerosis, 2016;246:334-337.

8. Kahe, F, Sharfaei, S, Pitliya, A, et al., Coronary artery tortuosity: a narrative review, Coron Artery Dis, 2020;31:187-192.

9. Jin, H, Ji, JJ, Zhu, Y, et al., Brain-Derived Neurotrophic Factor, a New Predictor of Coronary Artery Calcification, Clin Appl Thromb Hemost, 2021;27:1076029621989813.

10. Greenland, P, Bonow, RO, Brundage, BH, et al., ACCF/AHA 2007 clinical expert consensus document on coronary artery calcium scoring by computed tomography in global cardiovascular risk assessment and in evaluation of patients with chest pain: a report of the American College of Cardiology Foundation Clinical Expert Consensus Task Force (ACCF/AHA Writing Committee to Update the 2000 Expert Consensus Document on Electron Beam Computed Tomography) developed in collaboration with the Society of Atherosclerosis Imaging and Prevention and the Society of Cardiovascular Computed Tomography, J Am Coll Cardiol, 2007;49:378-402.

11. Khosravani-Rudpishi, M, Joharimoghadam, A and Rayzan, E, The significant coronary tortuosity and atherosclerotic coronary artery disease; What is the relation?, J Cardiovasc Thorac Res, 2018;10:209-213.

12. Lee, $A Y$, Han, $B$, Lamm, SD, et al., Effects of elastin degradation and surrounding matrix support on artery stability, Am J Physiol Heart Circ Physiol, 2012;302:H873-884. 
13. Han, HC and Fung, YC, Longitudinal strain of canine and porcine aortas, J Biomech, 1995;28:637641.

14. Dobrin, $\mathrm{PB}$, Schwarcz, TH and Baker, WH, Mechanisms of arterial and aneurysmal tortuosity, Surgery, 1988;104:568-571.

15. Han, HC, Twisted blood vessels: symptoms, etiology and biomechanical mechanisms, J Vasc Res, 2012;49:185-197.

16. Learoyd, BM and Taylor, MG, Alterations with age in the viscoelastic properties of human arterial walls, Circ Res, 1966;18:278-292.

17. Panwar, $P$, Hedtke, T, Heinz, A, et al., Expression of elastolytic cathepsins in human skin and their involvement in age-dependent elastin degradation, Biochim Biophys Acta Gen Subj, 2020;1864:129544.

18. Zarkovic, K, Larroque-Cardoso, P, Pucelle, M, et al., Elastin aging and lipid oxidation products in human aorta, Redox Biol, 2015;4:109-117.

19. Li, Y, Shi, Z, Cai, Y, et al., Impact of coronary tortuosity on coronary pressure: numerical simulation study, PLoS One, 2012;7:e42558.

20. Jakob, M, Spasojevic, D, Krogmann, ON, et al., Tortuosity of coronary arteries in chronic pressure and volume overload, Cathet Cardiovasc Diagn, 1996;38:25-31.

21. Choy, M, Ganesan, V, Thomas, DL, et al., The chronic vascular and haemodynamic response after permanent bilateral common carotid occlusion in newborn and adult rats, $\mathrm{J}$ Cereb Blood Flow Metab, 2006;26:1066-1075.

22. Chiha, J, Mitchell, P, Gopinath, B, et al., Gender differences in the prevalence of coronary artery tortuosity and its association with coronary artery disease, Int J Cardiol Heart Vasc, 2017;14:23-27.

23. Hutchins, GM, Bulkley, BH, Miner, MM, et al., Correlation of age and heart weight with tortuosity and caliber of normal human coronary arteries, Am Heart J, 1977;94:196-202.

24. Greenland, P, Blaha, MJ, Budoff, MJ, et al., Coronary Calcium Score and Cardiovascular Risk, J Am Coll Cardiol, 2018;72:434-447.

25. Ouimet, M, Barrett, TJ and Fisher, EA, HDL and Reverse Cholesterol Transport, Circ Res, 2019;124:1505-1518.

\section{Tables}

Table 1. General characteristics of patients included in the study 


\begin{tabular}{|c|c|c|c|c|}
\hline Characteristic & Total & N-CAT group & CAT group & $p$-value \\
\hline Participants (n) & 1280 & $835(65 \%)$ & $445(35 \%)$ & \\
\hline Gender & & & & $₫ 0.0001$ \\
\hline Male(n) & $658(51.4 \%)$ & $476(57 \%)$ & $182(40.9 \%)$ & \\
\hline Female (n) & $622(48.6 \%)$ & $359(43 \%)$ & $263(59.1 \%)$ & \\
\hline Age (years) & $62.3 \pm 12.7$ & $61.1 \pm 12.9$ & $65.2 \pm 11.8$ & $\varangle 0.0001$ \\
\hline $\mathrm{BMI}\left(\mathrm{kg} / \mathrm{m}^{2}\right)$ & & & & $₫ 0.0001$ \\
\hline $18.5-23.9$ & $412(31.2 \%)$ & $238(28.5 \%)$ & $174(39.1 \%)$ & \\
\hline $24-27.9$ & $635(49.6 \%)$ & $418(50.1 \%)$ & $217(48.8 \%)$ & \\
\hline$\llbracket 28$ & $233(18.2 \%)$ & $179(21.4 \%)$ & $54(12.1 \%)$ & \\
\hline Myocardial bridge & $555(43.4 \%)$ & $349(41.8 \%)$ & $206(46.3 \%)$ & 0.122 \\
\hline Diameter stenosis & & & & 0.204 \\
\hline No stenosis & $518(40.5 \%)$ & $351(42 \%)$ & $167(37.5 \%)$ & \\
\hline Slight stenosis & $207(16.2 \%)$ & $134(16.1 \%)$ & $73(16.4 \%)$ & \\
\hline Mild stenosis & $242(18.9 \%)$ & $153(18.3 \%)$ & $89(20 \%)$ & \\
\hline Moderate stenosis & $182(14.2 \%)$ & $107(12.8 \%)$ & $75(16.9 \%)$ & \\
\hline Severe stenosis & $131(10.2 \%)$ & $90(10.8 \%)$ & $41(9.2 \%)$ & \\
\hline Agatston score & & & & 0.111 \\
\hline 0 & $608(47.5 \%)$ & $411(49.2 \%)$ & $197(44.2 \%)$ & \\
\hline $1-100$ & $342(26.7 \%)$ & $226(27.1 \%)$ & $116(26.1 \%)$ & \\
\hline $101-400$ & $173(13.5 \%)$ & $101(12.1 \%)$ & $72(16.2 \%)$ & \\
\hline$₫ 400$ & $157(12.3 \%)$ & $97(11.6 \%)$ & $60(13.5 \%)$ & \\
\hline Smoking & $189(14.8 \%)$ & $131(15.7 \%)$ & $58(13 \%)$ & 0.202 \\
\hline Hypertension & $758(59.2 \%)$ & $474(56.8 \%)$ & $284(63.8 \%)$ & 0.014 \\
\hline Diabetes & $266(20.8 \%)$ & 178 (21.3\%) & $88(19.8 \%)$ & 0.517 \\
\hline \multicolumn{5}{|c|}{ Echocardiographic parameters } \\
\hline $\mathrm{AO}(\mathrm{cm})$ & $2.1 \pm 0.3$ & $2.2 \pm 0.3$ & $2.1 \pm 0.3$ & 0.006 \\
\hline $\mathrm{AAO}(\mathrm{cm})$ & $3.3 \pm 0.4$ & $3.3 \pm 0.4$ & $3.3 \pm 0.4$ & 0.577 \\
\hline $\mathrm{LA}(\mathrm{cm})$ & $3.8 \pm 0.6$ & $3.9 \pm 0.6$ & $3.8 \pm 0.6$ & 0.53 \\
\hline
\end{tabular}




\begin{tabular}{|lllll|}
\hline RVEDd $(\mathrm{cm})$ & $2.4 \pm 0.7$ & $2.4 \pm 0.3$ & $2.4 \pm 1.1$ & 0.771 \\
\hline IVS $(\mathrm{cm})$ & $1.1 \pm 0.2$ & $1.1 \pm 0.2$ & $1.1 \pm 0.3$ & 0.529 \\
\hline LVEDd $(\mathrm{cm})$ & $4.6 \pm 0.7$ & $4.7 \pm 0.6$ & $4.6 \pm 0.7$ & 0.002 \\
\hline LVPW $(\mathrm{cm})$ & $1.0 \pm 0.3$ & $1.0 \pm 0.3$ & $1.0 \pm 0.2$ & 0.216 \\
\hline Ejection Fraction $(\%)$ & $66 \pm 11.8$ & $65.8 \pm 11.8$ & $66.6 \pm 11.7$ & 0.239 \\
\hline Biochemical indexes & & & & \\
\hline Uric Acid (umol/l) & $341 \pm 103.2$ & $346.1 \pm 104.1$ & $331.6 \pm 101$ & 0.018 \\
\hline TG (mmol/l) & $1.6 \pm 1.2$ & $1.7 \pm 1.2$ & $1.5 \pm 1.2$ & 0.047 \\
\hline CHOL (mmol/l) & $4.5 \pm 1.0$ & $4.5 \pm 1.0$ & $4.6 \pm 1.1$ & 0.475 \\
\hline HDL (mmol/l) & $1.3 \pm 0.3$ & $1.3 \pm 0.3$ & $1.4 \pm 0.3$ & $\otimes 0.0001$ \\
\hline LDL (mmol/l) & $2.5 \pm 0.8$ & $2.6 \pm 0.8$ & $2.5 \pm 0.7$ & 0.496 \\
\hline ApoA1(g/l) & $1.2 \pm 0.3$ & $1.2 \pm 0.3$ & $1.2 \pm 0.3$ & 0.582 \\
\hline ApoB (g/l) & $0.9 \pm 0.2$ & $0.9 \pm 0.2$ & $0.9 \pm 0.2$ & 0.633 \\
\hline LPa (mg/l) & $124(59-296)$ & $118(58-274)$ & $138(61-328)$ & 0.117 \\
\hline
\end{tabular}

Table 2. Univariable and multivariable analysis of which factors are related to CAT 


\begin{tabular}{|c|c|c|c|c|}
\hline \multirow[b]{2}{*}{ Variable } & \multicolumn{2}{|l|}{ Univariable analysis } & \multicolumn{2}{|c|}{ Multivariable analysis } \\
\hline & Odds ratio $(95 \% \mathrm{Cl})$ & $p$-value & Odds ratio $(95 \% \mathrm{Cl})$ & $p$-value \\
\hline \multicolumn{5}{|l|}{ Gender } \\
\hline Male & Ref & & Ref & \\
\hline Female & $1.92(1.52-2.42)$ & $<0.0001$ & $1.60(1.24-2.17)$ & 0.001 \\
\hline Age (years) & $1.03(1.02-1.04)$ & $<0.0001$ & $1.02(1.01-1.03)$ & 0.003 \\
\hline \multicolumn{5}{|l|}{ BMI $\left(\mathrm{kg} / \mathrm{m}^{2}\right)$} \\
\hline $18.5-23.9$ & Ref & & Ref & \\
\hline $24-27.9$ & $0.71(0.55-0.92)$ & 0.009 & $0.77(0.59-1.02)$ & 0.064 \\
\hline $\mathbb{2} 28$ & $0.41(0.29-0.59)$ & $<0.0001$ & $0.47(0.32-0.70)$ & $<0.0001$ \\
\hline \multicolumn{5}{|l|}{ Diameter stenosis } \\
\hline No stenosis & Ref & & & \\
\hline Slight stenosis & $1.15(0.82-1.61)$ & 0.434 & $1.17(0.76-1.78)$ & 0.476 \\
\hline Mild stenosis & $1.22(0.89-1.68)$ & 0.218 & $0.93(0.60-1.46)$ & 0.764 \\
\hline Moderate stenosis & $1.47(1.04-2.09)$ & 0.029 & $1.01(0.60-1.70)$ & 0.974 \\
\hline Severe stenosis & $0.96(0.63-1.45)$ & 0.837 & $0.65(0.35-1.19)$ & 0.162 \\
\hline \multicolumn{5}{|l|}{ Agatston score } \\
\hline 0 & Ref & & & \\
\hline $1-100$ & $1.07(0.81-1.42)$ & 0.633 & $0.94(0.63-1.39)$ & 0.743 \\
\hline $101-400$ & $1.49(1.05-2.10)$ & 0.025 & $1.42(0.85-2.37)$ & 0.178 \\
\hline$\varangle 400$ & $1.29(0.89-1.86)$ & 0.17 & $1.23(0.69-2.19)$ & 0.482 \\
\hline Hypertension & $1.34(1.06-1.70)$ & 0.015 & $1.32(1.01-1.72)$ & 0.039 \\
\hline $\mathrm{AO}(\mathrm{cm})$ & $0.54(0.34-0.84)$ & 0.007 & $0.90(0.55-1.48)$ & 0.687 \\
\hline LVEDd (cm) & $0.74(0.61-0.90)$ & 0.002 & $0.89(0.73-1.10)$ & 0.309 \\
\hline Uric Acid (umol/I) & $0.99(0.97-1.00)$ & 0.019 & $1.00(0.99-1.00)$ & 0.868 \\
\hline $\mathrm{TG}(\mathrm{mmol} / \mathrm{l})$ & $0.90(0.81-1.00)$ & 0.049 & $0.98(0.88-1.10)$ & 0.741 \\
\hline $\mathrm{HDL}(\mathrm{mmol} / \mathrm{l})$ & $2.02(1.38-2.96)$ & $<0.0001$ & $1.45(0.96-2.21)$ & 0.081 \\
\hline
\end{tabular}

Table 3. Univariable and multivariable analysis of parameters directly affecting for CAT in female 


\begin{tabular}{|c|c|c|c|c|}
\hline & Univariable analysis & & Multivariable analys & \\
\hline Variable & Odds ratio $(95 \% \mathrm{Cl})$ & $p$-value & Odds ratio $(95 \% \mathrm{Cl})$ & $p$-value \\
\hline Age (years) & $1.03(1.01-1.04)$ & $<0.0001$ & $1.01(0.99-1.03)$ & 0.126 \\
\hline \multicolumn{5}{|l|}{$\mathrm{BMI}\left(\mathrm{kg} / \mathrm{m}^{2}\right)$} \\
\hline $18.5-23.9$ & Ref & & Ref & \\
\hline $24-27.9$ & $0.75(0.53-1.07)$ & 0.111 & $0.73(0.51-1.06)$ & 0.094 \\
\hline$\varangle 28$ & $0.49(0.31-0.81)$ & 0.005 & $0.51(0.31-0.84)$ & 0.008 \\
\hline \multicolumn{5}{|l|}{ Agatston score } \\
\hline 0 & Ref & & Ref & \\
\hline $1-100$ & $1.49(1.01-2.18)$ & 0.042 & $1.29(0.86-1.95)$ & 0.224 \\
\hline $101-400$ & $2.36(1.36-4.07)$ & 0.002 & $1.79(1.00-3.20)$ & 0.048 \\
\hline$\varangle 400$ & $1.82(1.07-3.11)$ & 0.028 & $1.33(0.72-2.44)$ & 0.358 \\
\hline Hypertension & $1.68(1.21-2.33)$ & 0.002 & $1.54(1.07-2.22)$ & 0.021 \\
\hline $\mathrm{HDL}(\mathrm{mmol} / \mathrm{l})$ & $1.88(1.12-3.18)$ & 0.018 & $1.86(1.07-3.24)$ & 0.028 \\
\hline
\end{tabular}

Table 4. Univariable and multivariable analysis of factors directly affecting for CAT in male

\begin{tabular}{|c|c|c|c|c|}
\hline \multirow[b]{2}{*}{ Variable } & \multicolumn{2}{|l|}{ Univariable analysis } & \multicolumn{2}{|c|}{ Multivariable analysis } \\
\hline & Odds ratio $(95 \% \mathrm{Cl})$ & $p$-value & Odds ratio $(95 \% \mathrm{Cl})$ & $p$-value \\
\hline Age (years) & $1.02(1.00-1.03)$ & 0.002 & $1.03(1.02-1.04)$ & $<0.0001$ \\
\hline \multicolumn{5}{|l|}{ BMI } \\
\hline $18.5-23.9$ & Ref & & Ref & \\
\hline $24-27.9$ & $0.77(0.52-1.13)$ & 0.175 & $0.72(0.56-0.94)$ & 0.014 \\
\hline$\otimes 28$ & $3.62(0.21-0.64)$ & $<0.0001$ & $0.46(0.32-0.66)$ & $<0.0001$ \\
\hline \multicolumn{5}{|l|}{ Diameter stenosis } \\
\hline No stenosis & Ref & & Ref & \\
\hline Slight stenosis & $1.61(0.98-2.64)$ & 0.06 & $1.05(0.74-1.50)$ & 0.769 \\
\hline Mild stenosis & $1.67(1.02-2.74)$ & 0.041 & $0.95(0.68-1.34)$ & 0.782 \\
\hline Moderate stenosis & $1.51(0.90-2.52)$ & 0.116 & $1.05(0.72-1.52)$ & 0.819 \\
\hline Severe stenosis & $1.01(0.56-1.82)$ & 0.937 & $0.67(0.43-1.04)$ & 0.071 \\
\hline
\end{tabular}


Figures

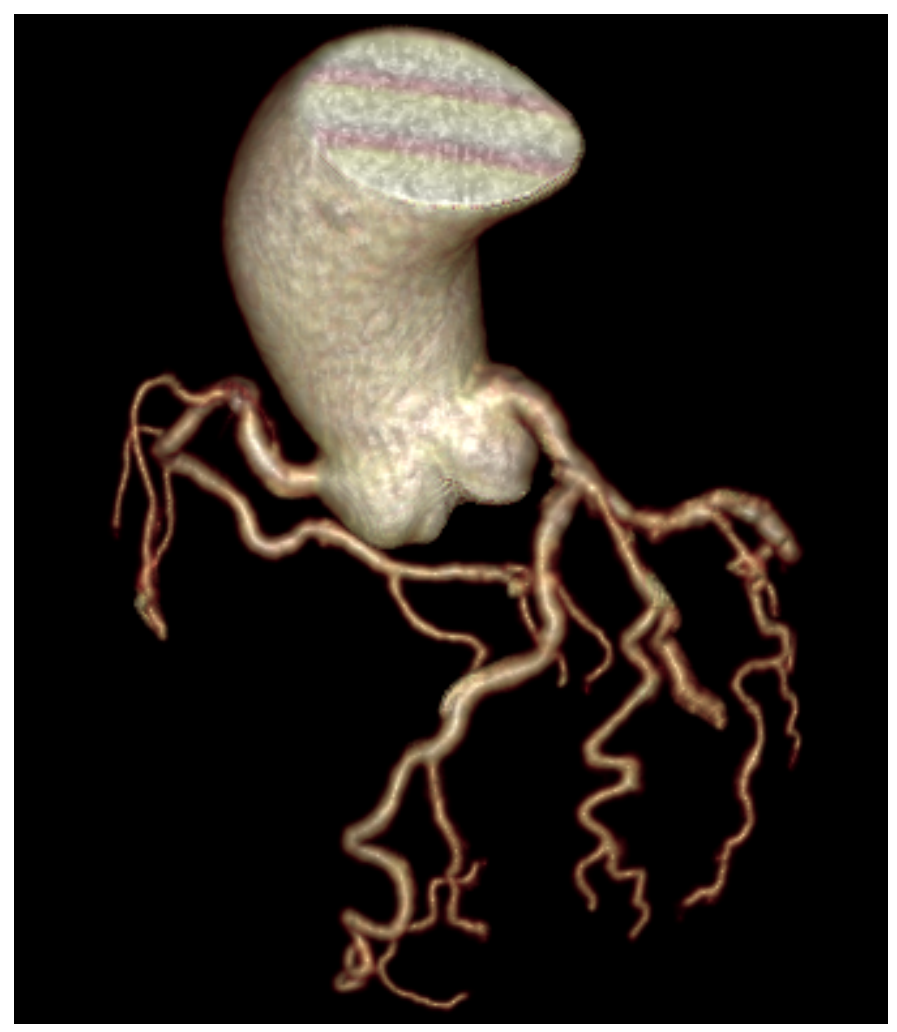

Figure 1

CTA shows tortuous coronary.

\section{Supplementary Files}

This is a list of supplementary files associated with this preprint. Click to download.

- Supplementary.docx 